\title{
Association between the nurse work environment and severe maternal morbidity in high-income countries: A systematic review protocol
}

Kyrah K Brown ( $\sim$ kyrah.brown@uta.edu )

University of Texas at Arlington https://orcid.org/0000-0002-7028-6791

Jessica G. Smith

University of Texas at Arlington

RaeAnna L Jeffers

University of Texas at Arlington

Claudy Jean Pierre

University of Texas at Arlington

\section{Protocol}

Keywords: nurse work environment, maternal morbidity, severe maternal morbidity, pregnancy complications

Posted Date: February 18th, 2020

DOI: https://doi.org/10.21203/rs.2.23905/v1

License: (c) (i) This work is licensed under a Creative Commons Attribution 4.0 International License. Read Full License 


\section{Abstract}

Background The United States has the highest maternal mortality and morbidity rates compared to its high-income peer nations. In high-income nations, a considerable amount of maternal morbidity cases are preventable and linked to provider-related factors. Better nurse work environments are associated with positive patient outcomes, but little is known about its impact on maternal morbidity. In this systematic review, we aim to identify the association between nurse work environment and maternal morbidity, specifically in high-income countries.

Methods This systematic review will include original articles on the association between nurse work environment and maternal morbidity. CINAHL, PubMed/Medline and the Cochrane Central Register of Controlled Trials will be searched to retrieve potential original articles that are published between 1990 and 2019 in English language. Citations will be screened by two reviewers, in two rounds, for inclusion based on a priori inclusion and exclusion criteria. Data extraction templates will be populated with data to evaluate the methodological and reporting quality of each study. A combination of structured narrative synthesis and quantitative summaries in tabular format will allow for discussion and recommendations for future research.

Discussion Results from this systematic review will provide evidence to elucidate the association between nurse work environment and maternal morbidity. While there is strong evidence demonstration the relation between nurse work environment and general patient outcomes, less is known about its influence on maternal morbidity. Findings from this review will help to guide research in the field and nursing professional in the development of targeted practices and policies aimed at reducing the rates of maternal morbidity.

\section{Background}

Maternal mortality, defined by the World Health Organization, refers to the "death of a woman while pregnant or within 42 days of termination of pregnancy, irrespective of the duration and site of the pregnancy, from any cause related to or aggravated by the pregnancy or its management but not from accidental or incidental causes." [1]. Each day, it is estimated that about 830 women die from preventable causes related to pregnancy and childbirth [1]. Maternal mortality, however, is only the 'tip of the iceberg' in terms of poor maternal health outcomes. Maternal morbidity is about 50 times more common than maternal death [2]. Severe maternal morbidity, defined as unexpected outcomes of labor and delivery that result in acute or long-term consequences to a woman's health during pregnancy through the standard postpartum period of 42 days [3]. The United States has the highest maternal morbidity rates compared to its high-income peer countries [4]. The rates of maternal mortality and morbidity in high-income peer countries declined substantially between 1990 and 2015 with the exception of the United States [4]. The United States lags far behind other high-income nations. In high-income countries, it is estimated that 
about $38 \%$ of severe maternal morbidity cases were preventable and $36 \%$ were not preventable but improvement in care was needed [5]. The most common preventable factors include provider-related factors (e.g., delays in diagnosis or treatment) [5, 6]. There is a growing body of work highlighting the potential influence of the health care system and standards of care on maternal health outcomes [7-10]

Nurses provide obstetrical care across the U.S. healthcare continuum, including in outpatient clinics and hospitals. Obstetrical nurses monitor for maternal condition changes in the outpatient setting and are responsible for coordinating timely care during obstetrical emergencies. Obstetrical nurses play a vital role in implementing standards of care, recognizing risk factors for complications, and communicating issues to maternal care providers in a timely and accurate manner[11]. The nurse work environment, defined as "the organizational characteristics of a work setting that facilitate or constrain professional nursing practice" [12], might be a cost-effective and modifiable nursing resource in healthcare to address the growing burden of maternal morbidity in high-income nations, especially the United States. Characteristics of the nurse work environment include nurse manager leadership, interprofessional relationships, nursing input into hospital decision-making and quality improvement, and adequate staffing [13]. Nurse work environments are an important global interest given their potential to be modified as a precursor to promote better patient outcomes. Better nurse work environments have been associated with better patient outcomes such as decreased mortalities in general, vascular, and orthopedic surgical patients and decreased rates for failure-to-rescue in large-scale studies [14].

There is a need to address existing knowledge gaps related to the potential role of the nurse work environment in contributing to the preventability of severe maternal morbidity. To our knowledge, there is no published systematic review that addresses our questions, which are as follows: (1) What is known about the association between nurse work environment and severe maternal morbidity in high-income countries? and (2) Are there specific dimensions of nurse work environment that may impact maternal morbidity in high-income countries? We have addressed our research questions through a comprehensive protocol targeting studies in this area from the last 30 years.

\section{Methods And Design}

\section{Design and registration}

The review protocol was submitted to the International Prospective Register of Systematic Reviews (PROSPERO) on April 24, 2019 (No. CRD42019124073). The protocol has been developed in accordance with the Preferred Reporting Items for Systematic Reviews and Meta-Analysis Protocols (PRISMA-P) [15] and the completed review will be reported in accordance with the PRISMA-P guidelines.

\section{Outcome}

The primary outcome of interest will be severe maternal morbidity, defined as unexpected outcomes of labor and delivery that result in acute or long-term consequences to a woman's health. Severe maternal morbidity indicators include acute myocardial infarction or aneurysm, acute renal failure, adult respiratory 
distress syndrome, cardiac arrest, shock, sepsis, hysterectomy, blood transfusions, eclampsia or preeclampsia, amniotic fluid embolism, severe anesthesia complications, cerebrovascular disorders, air and thrombotic embolism, and acute congestive heart failure or pulmonary edema. Articles of interest will examine associations between the construct of 'nurse work environment' and severe maternal morbidity operationalized as any of the previously mentioned conditions or operationalized generally as 'severe maternal morbidity'.

\section{Search strategy}

An extensive literature review will be performed and refined using the following citation databases: PubMed/Medline, CINAHL, Scopus, Google Scholar, and Cochrane Library. Searches will be limited to literature published from 1990 onwards. Between 1990 and 2015, the global rates of maternal mortality and morbidity declined drastically [1]. Therefore, the authors reason that any the availability of published literature on this topic would be available after 1990. The search strategy was developed iteratively based on the authors' combined expertise in the topic areas and in systematic review. The search strategy will be tested for sensitivity and specificity by calculating the number of relevant results divided by the total number of reports. Specificity is calculated as the number of relevant reports identified divided by the total number of reports identified.

\section{Data screening}

Once all database searches have been completed, citations will be exported to the reference manager Endnote $\mathrm{X} 7$ and duplicates removed. Remaining citations will be exported into a formatted Microsoft Office Excel ${ }^{\mathrm{TM}}$ spreadsheet and screened by two reviewers for inclusion using predetermined inclusion and exclusion criteria described in the following section of this protocol. The first phase of screening will assess eligibility based on title and abstract. The second phase of screening will assess the eligibility of all remaining studies based on a full-text review. All abstracts and full texts will be sourced as necessary. Disagreements will be resolved through discussion between authors. During the second phase of screening, the reviewers will document the reasons for exclusion decision. A PRISMA-P diagram will be generated to document the study selection process.

\section{Inclusion and exclusion criteria}

The inclusion and exclusion criteria for this review are presented in Table 1. High-income country were identified using the World Bank classification which categorizes nations based on gross national income per capita [16]. Countries were included if they were classified as high-income consistently in the last 5 years. 
Table 1

Review inclusion and exclusion criteria

Inclusion criteria

Articles assessing the association between at least one of the dimensions of nurse work environment and maternal patient outcomes

Articles describing research conducted in a highincome country ${ }^{1}$ (e.g., United States, Canada, United Kingdom, etc.)

Articles that focus on a unit of analysis at the site/hospital or population level

Articles published between 1990 and 2019

Articles published in English language

Original research articles describing descriptive studies, cohort studies, non-randomized interventions

\section{Exclusion criteria}

Articles assessing the association between nurse work environment and nurse, nursing or unit outcomes

Articles describing research conducted in a lowor middle-income country

Articles published before 1990

Articles published in languages other than English

Articles for which the full methods and results are not readily available.

Case reports, systematic reviews, guidelines, conference papers, poster presentations, commentaries, editorials, or opinion pieces

\section{Data extraction}

The two reviewers will independently extract data from the full texts of literature and populate the extracted data into a formatted Microsoft Office Excel spreadsheet. This form will be piloted on two studies independently by two reviewers and compared to ensure consistency in the interpretation of the data being extracted and to test the functioning of the formatted cells. Data will be collected as follows: author, study, study type, objectives, design, setting type, location (country) population sample size, standardized measurement tool used (if any), patient/maternal outcome, and key finding. The reviewers will review the collected data. The data extracted will include all details specific to the review objectives. Data extraction will be cross-checked independently.

\section{Quality assessment (Risk of Bias Assessment)}

The quality of reporting and risk for bias will be assessed for each article included in the final sample. Two reviewers (KB and JS) will assess quality of reporting and risk for bias independently using the Cochrane Risk of Bias Assessment Tool for Non-Randomized Studies of Interventions (ROBIN-I) and the Strengthening the Reporting of Observational Studies in Epidemiology (STROBE) Checklist. Observational studies will be evaluated in accordance with the ROBIN-I [13] and their quality of reporting will be assessed using the STROBE Checklist [14]. The included studies are expected to be all observational studies with no randomized controlled trials (RCTs). If any RCTs emerge, they will be assessed using the Cochrane Handbook "Risk of Bias" assessment tool [15]. Disagreements will be resolved by consensus or involvement of a third reviewer if indicated. 


\section{Meta-bias}

To reduce risk for publication bias for this systematic review, published peer-reviewed literature, grey literature, and proceedings for national and international conferences will be searched. Selective reporting of outcomes in all included studies will be assessed to determine whether outcomes that were planned were actually reported in the published studies.

\section{Data Synthesis}

In order to ensure a robust and transparent synthesis of evidence, the guidance on the conduct of narrative synthesis in systematic reviews will be used to conduct this review's narrative synthesis [16]. The guidance offers four elements, along with several techniques that may be applied in the synthesis process. The four elements alongside the preliminary narrative synthesis plan are presented in Table 2 . We will use the Grading of Recommendations Assessment, Development and Evaluation (GRADE) which includes items related to risk of bias, imprecision, inconsistency, indirectness and publication bias. This tool will be used to rate the overall evidence and develop recommendations based on the available evidence. 
Table 2

Narrative Synthesis Plan

\section{Guidance Preliminary Plan Elements}

1. Developing a theory

2. Developing a preliminary synthesis
The basic theory underlying this review is that by identifying associations between SMM and nurse work environment, researchers and healthcare providers can design and test interventions to address SMM.

The tools and techniques that will be used included drawing of tables, textual descriptions of studies, and transforming data for comparison. The tables will be organized according to the setting/context, type of subjects involved, study design, results of study quality assessment, use of a validated nurse work environment measure, and outcome measures. It is possible that objective and nurse-reported outcomes will emerge. In order to facilitate comparison and interpretation, the data will be clustered based on those characteristics. Subsequently, textual descriptions will help draw out and report important aspects of the studies shown in the tables.

3. Exploring relationships within and between studies
The techniques used at this stage will include qualitative case descriptions. The processes described above will help assess similarities and differences between studies. Differences in studies will be discussed by location (country), type of facility, and other relevant sub-categories. By comparing and contrasting, we will explore how factors such as study design, population characteristics, and context may explain the study results. Building upon the initial textual descriptions, key aspects of the included studies will be qualitatively described comprehensively and interpreted to enhance understanding of any discrepancies between studies.
4. Assessing the robustness of the synthesis
At this stage, validity assessment and critical reflection on the synthesis process will be employed. As explained above, assessment of the methodological quality of the primary studies included in the review forms part of the data extraction process and therefore occurs at an earlier stage. Additionally, the narrative synthesis process will be critically reflected upon. At the end of the review, the exact process applied will be reported in the final paper.

\section{Potential limitations}

A potential limitation is the likelihood that there will be great heterogeneity across the included studies with respect to differences in measurement instruments used, differences in reported outcomes, and differences in patient or hospital characteristics. Should this issue arise it will be discussed thoroughly in the narrative synthesis.

\section{Protocol amendments}

Any amendments to the protocol will be outlined in an addendum made to specify and justify changes. These modifications and their justifications will also be included in the final report.

\section{Discussion}

This systematic review will provide evidence to elucidate the association between nurse work environment and maternal morbidity. While there is strong evidence demonstrating the association between nurse work environment on general patient outcomes, less is known about the influence on 
maternal morbidity. To the best of our knowledge, this systematic review will be the first to address this question. This review is timely, not only as it fills a gap in the current literature, but also because political and clinical attention to the issue of maternal mortality and morbidity is increasing, particularly in the United States. The findings from this review will help to guide nursing professionals and researchers in the development of targeted practices and policies aimed at reducing the rates of maternal morbidity, and eventually maternal mortality.

\section{Abbreviations}

AACN: American Association of Colleges of Nursing ; PROSPERO: International Prospective Register of Systematic Reviews; PRISMA-P: Preferred Reporting Items for Systematic review and Meta-Analysis Protocols ; CINAHL: Cumulative Index of Nursing and Allied Health Literature; ROBIN-I: Risk of Bias in Non-randomized Studies of Interventions ; STROBE: Strengthening The Reporting of Observational Studies in Epidemiology

\section{Declarations}

\section{Ethics approval and consent to participate}

This systematic review protocol was registered with the International Prospective Registry of Systematic Reviews (POSPERO) on April 24, 2019. Any amendments to the protocol will be documented on the PROSPERO site. Ethical approval is not required for this study as it is a systematic review. The results will be submitted to peer-reviewed journals and presented at national meetings.

\section{Consent for publication}

Not applicable

\section{Availability of data and materials}

Not applicable

\section{Competing interests}

The authors declare that they have no competing interests.

\section{Funding}

This work was supported by start-up funds from the College of Nursing and Health Innovation at the University of Texas at Arlington.

\section{Authors' contributions}


KB and JS conceptualized the study. KB, JS, and RJ contributed to the design of the study. The protocol was drafted by KB, with JS, RJ, and CJP providing critical revisions for intellectual content. RJ will perform database searches and extract data for screening. KB and JS will independently screen studies for eligibility in this systematic review, and RJ will serve as a third reviewer to resolve disagreements in screening. KB, JS, RJ, and CJP will assess for the risk of bias during data synthesis. KB, JS, RJ, and CJP meet the criteria for authorship and have approved the final article and that all those entitled to authorship are listed as authors.

\section{Acknowledgements}

We would like to express our gratitude to Peace Ossom Williamson, Director for Research Data Services at the University of Texas at Arlington for her support and expertise.

\section{Authors' information}

${ }^{1}$ Assistant Professor, Department of Kinesiology, College of Nursing and Health Innovation, University of Texas at Arlington, Arlington, TX, United States. ${ }^{2}$ Assistant Professor, College of Nursing and Health Innovation, University of Texas at Arlington, Arlington, TX, United States. ${ }^{3}$ Health Sciences Librarian, University of Texas at Arlington, Arlington, TX, United States. ${ }^{4}$ Graduate Research Assistant, Department of Kinesiology, College of Nursing and Health Innovation, University of Texas at Arlington, Arlington, TX, United States.

\section{References}

1. World Health Organization, UNICEF, United Nations, Department of Economic and Social Affairs, Population Division, World Bank. Trends in maternal mortality: 1990 to 2015: estimates by WHO, UNICEF, UNFPA, World Bank Group and the United Nations Population Division [Internet]. 2015 [cited 2018 Nov 8]. Available from:

http://www.who.int/reproductivehealth/publications/monitoring/maternal-mortality-2015/en/

2. Callaghan WM, Mackay AP, Berg CJ. Identification of severe maternal morbidity during delivery hospitalizations, United States, 1991-2003. Am J Obstet Gynecol. 2008 Aug;199(2):133.e1-8.

3. Reed H, Koblinsky MA, Mosley WH. The Consequences of Maternal Morbidity and Maternal Mortality: Report of a Workshop [Internet]. Washington, D.C.: National Academies Press; 2000 [cited 2020 Jan 17]. Available from: http://www.nap.edu/catalog/9800

4. Kassebaum NJ, Barber RM, Bhutta ZA, Dandona L, Gething PW, Hay SI, et al. Global, regional, and national levels of maternal mortality, 1990-2015: a systematic analysis for the Global Burden of Disease Study 2015. The Lancet. 2016 Oct;388(10053):1775-812.

5. Lawton B, MacDonald EJ, Brown SA, Wilson L, Stanley J, Tait JD, et al. Preventability of severe acute maternal morbidity. Am J Obstet Gynecol. 2014 Jun;210(6):557.e1-6. 
6. Geller SE, Koch AR, Garland CE, MacDonald EJ, Storey F, Lawton B. A global view of severe maternal morbidity: moving beyond maternal mortality. Reproductive Health. 2018 Jun 22;15(1):98.

7. Kozhimannil KB, Thao V, Hung P, Tilden E, Caughey AB, Snowden JM. Association between Hospital Birth Volume and Maternal Morbidity among Low-Risk Pregnancies in Rural, Urban, and Teaching Hospitals in the United States. Am J Perinatol. 2016;33(6):590-9.

8. Attanasio L, Kozhimannil KB. Patient-reported communication quality and perceived discrimination in maternity care. Med Care. 2015 Oct;53(10):863-71.

9. Howell EA, Zeitlin J. Improving hospital quality to reduce disparities in severe maternal morbidity and mortality. Semin Perinatol. 2017;41(5):266-72.

10. Howell EA, Egorova N, Balbierz A, Zeitlin J, Hebert PL. Black-white differences in severe maternal morbidity and site of care. Am J Obstet Gynecol. 2016 Jan;214(1):122.e1-7.

11. Morton $\mathrm{CH}$, VanOtterloo LR, Seacrist MJ, Main EK. Translating Maternal Mortality Review Into Quality Improvement Opportunities in Response to Pregnancy-Related Deaths in California. Journal of Obstetric, Gynecologic \& Neonatal Nursing. 2019 May;48(3):252-62.

12. Lake ET. Development of the practice environment scale of the nursing work index. Res Nurs Health. 2002 Jun;25(3):176-88.

13. Lake ET, Sanders J, Duan R, Riman KA, Schoenauer KM, Chen Y. A Meta-Analysis of the Associations Between the Nurse Work Environment in Hospitals and 4 Sets of Outcomes: Medical Care. 2019 May;57(5):353-61.

14. Aiken LH, Clarke SP, Sloane DM, Lake ET, Cheney T. Effects of Hospital Care Environment on Patient Mortality and Nurse Outcomes: JONA: The Journal of Nursing Administration. 2008 May;38(5):2239.

15. Shamseer L, Moher D, Clarke M, Ghersi D, Liberati A, Petticrew M, et al. Preferred reporting items for systematic review and meta-analysis protocols (PRISMA-P) 2015: elaboration and explanation. BMJ. 2015 Jan 2;349(jan02 1):g7647-g7647.

16. World Bank. World Bank Country and Lending Groups - World Bank Data Help Desk [Internet]. World Bank Country and Lending Groups. 2018 [cited 2020 Feb 7]. Available from: https://datahelpdesk.worldbank.org/knowledgebase/articles/906519-world-bank-country-andlending-groups

17. Sterne JA, Hernán MA, Reeves BC, Savović J, Berkman ND, Viswanathan M, et al. ROBINS-I: a tool for assessing risk of bias in non-randomised studies of interventions. BMJ. 2016 Oct 12;i4919.

18. von Elm E, Altman DG, Egger M, Pocock SJ, Gøtzsche PC, Vandenbroucke JP. The Strengthening the Reporting of Observational Studies in Epidemiology (STROBE) statement: guidelines for reporting observational studies. Journal of Clinical Epidemiology. 2008 Apr;61(4):344-9.

19. Higgins JPT, Altman DG, Gotzsche PC, Juni P, Moher D, Oxman AD, et al. The Cochrane Collaboration's tool for assessing risk of bias in randomised trials. BMJ. 2011 Oct 18;343(oct18 2):d5928-d5928. 
20. Popay J, Roberts H, Sowden A, Petticrew M, Arai L, Rodgers M, et al. Guidance on the conduct of narrative synthesis in systematic reviews: A product from the ESRC Methods Programme [Internet]. Lancaster University; 2006 [cited 2020 Jan 17]. Available from: http://rgdoi.net/10.13140/2.1.1018.4643

\section{Supplementary Files}

This is a list of supplementary files associated with this preprint. Click to download.

- PRISMAPchecklistFINAL.pdf 